TRANSACTIONS OF THE

AMERICAN MATHEMATICAL SOCIETY

Volume 350, Number 11, November 1998, Pages 4693-4708

S 0002-9947(98)02266-1

\title{
MODEL ASPHERICAL MANIFOLDS WITH NO PERIODIC MAPS
}

\author{
WIM MALFAIT
}

\begin{abstract}
A. Borel proved that, if the fundamental group $E$ of an aspherical manifold $M$ is centerless and the outer automorphism group of $E$ is torsionfree, then $M$ admits no periodic maps, or equivalently, there are no non-trivial finite groups of homeomorphisms acting effectively on $M$. In the literature, taking off from this result, several examples of (rather complex) aspherical manifolds exhibiting this total lack of periodic maps have been presented.

In this paper, we investigate to what extent the converse of Borel's result holds for aspherical manifolds $M$ arising from Seifert fiber space constructions. In particular, for e.g. flat Riemannian manifolds, infra-nilmanifolds and infrasolvmanifolds of type (R), it turns out that having a centerless fundamental group with torsion-free outer automorphism group is also necessary to conclude that all finite groups of affine diffeomorphisms acting effectively on the manifold are trivial. Finally, we discuss the problem of finding (less complex) examples of such aspherical manifolds with no periodic maps.
\end{abstract}

\section{Preliminaries}

Let us start with some notational remarks used throughout this paper. If $G$ is a group, $\operatorname{Aut}(G)$ denotes the group of automorphisms of $G$. If $g$ is an element of $G$, we write $\mu(g)$ for the conjugation in $G$ determined by $g$; i.e. $\mu(g)(x)=g x g^{-1}$. Then $\mu(G)$ is the inner automorphism group, $\operatorname{Inn}(G)$, and $\operatorname{Out}(G)=\operatorname{Aut}(G) / \operatorname{Inn}(G)$ is the outer automorphism group of $G$. Write $p: \operatorname{Aut}(G) \rightarrow \operatorname{Out}(G)$ for the natural projection. A group $G$ is said to be complete if it has trivial center $Z(G)$ and all its automorphisms are inner; i.e. $\operatorname{Out}(G)=\{1\}=Z(G)$ and $\operatorname{Aut}(G) \cong G$.

For a normal subgroup $N$ of $G, \operatorname{Aut}(G, N)$ denotes the group of all $G$-automorphisms mapping $N$ into itself and $\operatorname{Out}(G, N)=\operatorname{Aut}(G, N) / \operatorname{Inn}(G)$. Of course, if $N$ is characteristic in $G$, $\operatorname{Aut}(G, N)=\operatorname{Aut}(G)$ and $\operatorname{Out}(G, N)=\operatorname{Out}(G)$.

For a subset $X$ in $G, C_{G} X$ is the centraliser and $N_{G} X$ the normaliser of $X$ in $G$. $X$ is called self-normalising in $G$ if and only if $X$ coincides with its normaliser in $G$. If $G$ acts on a set $X, X^{G}$ denotes the subset of $X$ consisting of all elements fixed under the action of $G$.

Each group extension $1 \rightarrow N \rightarrow E \stackrel{j}{\rightarrow} F \rightarrow 1$ induces, by choosing a normalised section $s: F \rightarrow E(j \circ s$ is the identity and $s(1)=1)$ and via conjugation in $E$, a

Received by the editors December 19, 1996.

1991 Mathematics Subject Classification. Primary 57S25, 20F34, 20H15.

Key words and phrases. Aspherical manifold, periodic map, Seifert fiber space construction, infra-nil- and infra-solvmanifold of type (R).

The author is a Postdoctoral Fellow of the Fund for Scientific Research - Flanders (Belgium) (F.W.O.). 
map (not necessarily a homomorphism!) $\varphi: F \rightarrow \operatorname{Aut}(N)$ sending $x \in F$ to a $N$ automorphism $\varphi(x): n \mapsto s(x) n s(x)^{-1}(n \in N)$. This induces a homomorphism $\psi: F \stackrel{\varphi}{\rightarrow} \operatorname{Aut}(N) \stackrel{p}{\rightarrow} \operatorname{Out}(N)$ which we call an abstract kernel. We say that $1 \rightarrow N \rightarrow E \rightarrow F \rightarrow 1$ realises, or is compatible with, $\psi$. In the sequel we will refer to the following lemma:

Lemma 1.1. If a group extension $1 \rightarrow N \rightarrow E \rightarrow F \rightarrow 1$ realises an injective abstract kernel, then $Z(N)^{F}=Z(E)$.

Proof. Fix a section $s: F \rightarrow E$, inducing a map $\varphi: F \rightarrow \operatorname{Aut}(N)$ (and a welldefined group action of $F$ on $Z(N)$ ) and an injective abstract kernel $\psi: F \rightarrow$ $\operatorname{Out}(N)$. Assume $n_{0} \in Z(N)^{F}$. Take an element $e=n s(x)=(n, x)$ in $E(n \in N$, $x \in F)$. Then

$$
(n, x)\left(n_{0}, 1\right)=\left(n \cdot \varphi(x)\left(n_{0}\right), x\right)=\left(n \cdot n_{0}, x\right)=\left(n_{0} \cdot n, x\right)=\left(n_{0}, 1\right)(n, x) .
$$

Hence $n_{0} \in Z(E)$. Conversely, assume $\left(n_{0}, x_{0}\right) \in Z(E)$ and take $n \in N$. Then $\left(n_{0}, x_{0}\right)(n, 1)=\left(n_{0} \cdot \varphi\left(x_{0}\right)(n), x_{0}\right)$ and $(n, 1)\left(n_{0}, x_{0}\right)=\left(n \cdot n_{0}, x_{0}\right)$ or $\varphi\left(x_{0}\right)=\mu\left(n_{0}^{-1}\right)$. Hence $x_{0}$ belongs to the kernel of $\psi$. Since $\psi$ is injective, it follows that $x_{0}=1$. Moreover, for each $x \in F,\left(n_{0}, 1\right)(1, x)=\left(n_{0}, x\right)$ and $(1, x)\left(n_{0}, 0\right)=\left(\varphi(x)\left(n_{0}\right), x\right)$ or $n_{0} \in Z(N)^{F}$.

An extension $1 \rightarrow N \rightarrow E \rightarrow F \rightarrow 1$ is called admissible ([LR81]) if and only if $C_{E} N$ is torsion-free. For later use, we note that

Lemma 1.2. An extension $1 \rightarrow N \rightarrow E \rightarrow F \rightarrow 1$ realising an injective abstract kernel is admissible if and only if $Z(N)$ is torsion-free.

Proof. The reader only has to verify that the kernel of the abstract kernel induced by $1 \rightarrow N \rightarrow E \rightarrow F \rightarrow 1$ is isomorphic to $C_{E} N / Z(N)$.

Lemma 1.3. Let $G$ be a group and $1 \rightarrow N \rightarrow E \stackrel{j}{\rightarrow} F \rightarrow 1$ an admissible group extension. If $f^{\prime}: E \rightarrow G$ is a group homomorphism such that its restriction $\left.f^{\prime}\right|_{N}$ to $N$ is injective and $j\left(\operatorname{Ker}\left(f^{\prime}\right)\right)$ is finite, then $f^{\prime}$ itself is injective.

Proof. Obviously, an element $x$ in the kernel of $f^{\prime}$ is a torsion element of $E$, since $j\left(\operatorname{Ker}\left(f^{\prime}\right)\right)$ is finite and $\left.f^{\prime}\right|_{N}$ is injective. Moreover, for each $n \in N$,

$$
\left.f^{\prime}\right|_{N}\left(x n x^{-1}\right)=f^{\prime}\left(x n x^{-1}\right)=f^{\prime}(n)=\left.f^{\prime}\right|_{N}(n)
$$

or $x \in C_{E} N$ (since $\left.f^{\prime}\right|_{N}$ is injective). Hence, since $1 \rightarrow N \rightarrow E \rightarrow F \rightarrow 1$ is admissible, $x=1$.

\section{MODEL ASPHERICAL MANIFOLDS}

\section{AND THEIR FIBER PRESERVING HOMEOMORPHISMS}

An aspherical manifold $M$ is a closed, connected manifold whose universal covering is contractible. The starting point for the study of these spaces was the work of Hurewicz ([Hur36]). An aspherical manifold is a $K(E, 1)$-space: all higher homotopy groups are trivial, but the fundamental group $\pi_{1}(M) \cong E$ ([Bro82, §I.4]). An interesting class of aspherical manifolds are those arising from Seifert fiber space constructions, sometimes called model aspherical manifolds. Let us review the main aspects of this method (see also [KLR83], [Mal96], ...).

Assume $L$ is a connected and simply connected Lie group. A discrete subgroup $N$ of $L$ is a lattice of $L$ if and only if $N$ is cocompact (that is, $N \backslash L$ is compact) 
For a lattice $N$ of $L$, we say that $(N, L)$ has the Unique Automorphism Extension Property (UAEP) if and only if each automorphism of $N$ extends uniquely to an automorphism of $L$. Consequently, there is an inclusion $\operatorname{Aut}(N) \rightarrow \operatorname{Aut}(L)$ and a morphism $\operatorname{Out}(N) \rightarrow \operatorname{Out}(L)$.

Assume $W$ is a reasonable (e.g. connected, simply connected, paracompact ANR) topological space and such that $L \times W$ is a manifold. Write $\mathcal{H}(W)$ for the homeomorphism group of $W$ and $\mathcal{M}\left(W, L^{*}\right)$ for the group of continuous mappings $W \rightarrow L$ with multiplication: for $\lambda_{1}, \lambda_{2} \in \mathcal{M}\left(W, L^{*}\right)$ and for each $w \in W$, $\left(\lambda_{1} * \lambda_{2}\right)(w)=\lambda_{2}(w) \cdot \lambda_{1}(w)$. Then $\operatorname{Aut}(L) \times \mathcal{H}(W)$ acts on $\mathcal{M}\left(W, L^{*}\right)$ as follows: if $(g, h) \in \operatorname{Aut}(L) \times \mathcal{H}(W)$ and $\lambda \in \mathcal{M}\left(W, L^{*}\right)$, then ${ }^{(g, h)} \lambda=g \circ \lambda \circ h^{-1}$. The corresponding semidirect product $\mathcal{M}\left(W, L^{*}\right) \rtimes(\operatorname{Aut}(L) \times \mathcal{H}(W))=\mathcal{H}^{f}(L \times W)$, with group law $(\lambda, g, h)\left(\lambda^{\prime}, g^{\prime}, h^{\prime}\right)=\left(\left(g \circ \lambda^{\prime} \circ h^{-1}\right) \cdot \lambda, g g^{\prime}, h h^{\prime}\right)$, is a transformation group of the product space $L \times W$, i.e. if $(x, w) \in L \times W$, then ${ }^{(\lambda, g, h)}(x, w)=$ $(g(x) \cdot \lambda(h(w)), h(w))$. Clearly, each element of $\mathcal{H}^{f}(L \times W)$ preserves the fibers of the principal fibering $L \rightarrow L \times W \rightarrow W$. The Lie group $L$ can be seen as a subgroup of $\mathcal{M}\left(W, L^{*}\right) \rtimes \operatorname{Inn}(L)$ (via the embedding $l: L \rightarrow \mathcal{H}^{f}(L \times W): x \rightarrow(x, \mu(x), 1)$ ) and acts on $L \times W$ as (left) translations in the $L$-factor. In fact, $\mathcal{H}^{f}(L \times W)$ is the normaliser of $l(L)$ in the homeomorphism group $\mathcal{H}(L \times W)$ of $L \times W$. Therefore, $\mathcal{H}^{f}(L \times W)$ is referred to as the group of fiber preserving self-homeomorphisms of $L \times W$ compatible with left translations.

We say that a properly discontinuous action $\rho: F \rightarrow \mathcal{H}(W)$ of a group $F$ on $W$ has the $L$-Seifert property if and only if for every extension $1 \rightarrow L \rightarrow \mathcal{E} \rightarrow F \rightarrow 1$ (inducing an abstract kernel $\psi: F \rightarrow \operatorname{Out}(L)$ ), there exists a homomorphism $\hat{\Psi}$ making the following diagram commutative:

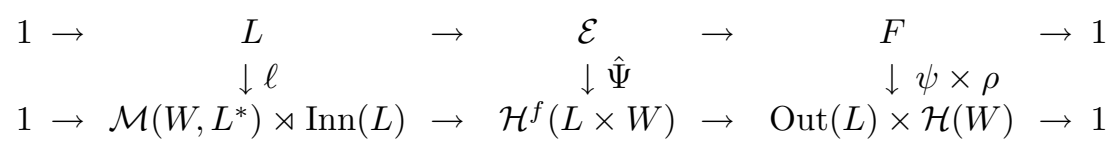

and such that $\hat{\Psi}$ is unique up to conjugation by elements of $\mathcal{M}\left(W, L^{*}\right)$.

A quadruple $(N, E, \rho, W)$ is called an $L$-set of data if and only if $N$ is a lattice in $L$ such that $(N, L)$ has UAEP and $N$ is a normal subgroup of $E$ such that $\rho: F=E / N \rightarrow \mathcal{H}(W)$ is a properly discontinuous action of $F$ on $W$ having the $L$-Seifert property. For example, if $L$ is nilpotent and $L$ contains a lattice $N$, then for every extension $1 \rightarrow N \rightarrow E \rightarrow F \rightarrow 1$ and every properly discontinuous action $\rho$ of $F$ on $W,(N, E, \rho, W)$ is an $L$-set of data ([KLR83]). If $L$ is solvable, with connected center and if $N$ is a lattice in $L$ such that $(N, L)$ has UAEP, then for every extension $1 \rightarrow N \rightarrow E \rightarrow F \rightarrow 1$ and every properly discontinuous action $\rho$ of $F$ on $W,(N, E, \rho, W)$ is an $L$-set of data ([LR89, Thm. 3]).

For an $L$-set of data $(N, E, \rho, W)$, a Seifert construction is a homomorphism $\Psi: E \rightarrow \mathcal{H}^{f}(L \times W)$ making the following diagram commutative:

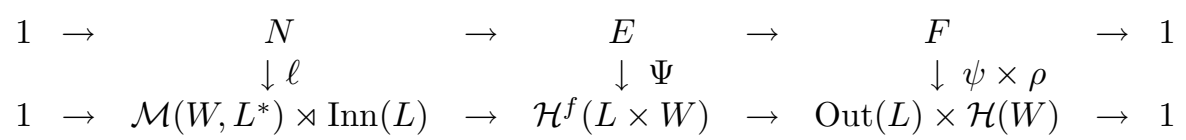

(notice that $\psi: F \rightarrow \operatorname{Out}(L)$ is well determined by the extension $1 \rightarrow N \rightarrow$ $E \rightarrow F \rightarrow 1$ and the UAEP of $(N, L))$. This Seifert construction concept has the following three important properties: 
Existence. For each $L$-set of data $(N, E, \rho, W)$, there exists a Seifert construction (here the UAEP of $(N, L)$ is crucial).

Uniqueness-1. Let $(N, E, \rho, W)$ be an $L$-set of data and fix an embedding $\epsilon$ : $N \rightarrow L$ of $N$ in $L$. Then, a Seifert construction $\Psi$ with respect to this embedding is unique up to conjugation by elements of $\mathcal{M}\left(W, L^{*}\right)$.

Uniqueness-2. Assume $\hat{g} \in \operatorname{Aut}(N)$. Let $\Psi$ and $\Psi^{\prime}$ be Seifert constructions for the same $L$-set of data $(N, E, \rho, W)$, but corresponding resp. to embeddings $\epsilon, \epsilon^{\prime}=$ $\epsilon \circ \hat{g}: N \rightarrow L$. Then (because of the UAEP of $(N, L)$ ), there exists a unique $g \in \operatorname{Aut}(L)$ such that $\epsilon^{\prime}=g \circ \epsilon$ and $\Psi^{\prime}=\mu(\lambda, g) \circ \Psi$, for a $\lambda \in \mathcal{M}\left(W, L^{*}\right)$.

Rigidity. Let $(N, E, \rho, W)$ be an $L$-set of data. Assume $\theta: E \rightarrow E$ is an automorphism inducing automorphisms $\hat{\theta}: N \rightarrow N$ and $\bar{\theta}: F \rightarrow F$ such that there exists a homeomorphism $h \in \mathcal{H}(W)$ for which $\mu(h) \circ \rho=\rho \circ \bar{\theta}$. Then, for each Seifert construction $\Psi$ for this set of data, there exists a fiber preserving homeomorphism $\hat{h} \in \mathcal{H}^{f}(L \times W)$ such that $\Psi \circ \theta=\mu(\hat{h}) \circ \Psi$.

The action of $E$ on $L \times W$, via a Seifert construction $\Psi: E \rightarrow \mathcal{H}^{f}(L \times W)$ associated to a $L$-set of data $(N, E, \rho, W)$, is properly discontinuous. Moreover, if $(N, E, \rho, W)$ is an admissible set of data (that is, $1 \rightarrow N \rightarrow E \rightarrow F \rightarrow 1$ is admissible), then, because of Lemma 1.3 (note that the kernel of $\rho$ is finite), $E$ acts effectively on $L \times W$ (via $\Psi$ ). Remark also that $E$ acts with compact quotient if and only if $F$ acts with compact quotient on $W$. So, if $W$ and $L$ are contractible, $F \backslash W$ is compact and if $E$ is torsion-free, then the given set of data determines a $K(E, 1)$-manifold $M=E \backslash(L \times W)$. We refer to this Seifert fiber space as the model aspherical manifold (with typical fiber $N \backslash L$ and $F \backslash W$ as its base) associated to the $L$-set of data $(N, E, \rho, W)$.

Many families of aspherical manifolds arising in this way, from an $L$-set of data $(N, E, \rho, W)$, have been studied in the literature before. We refer to

- [LR81] for flat Riemannian manifolds: $L=\mathbb{R}^{k}\left(k \in \mathbb{N}_{0}\right), N=\mathbb{Z}^{k}$ is of finite index in $E$ ( $E$ is a Bieberbach group), $W$ is a point and $\rho$ is the trivial action,

- [KLR83] for infra-nilmanifolds: $L$ is nilpotent, $N$ is of finite index in $E$ ( $E$ is an almost-Bieberbach group), $W$ is a point and $\rho$ is the trivial action,

- [Lee95] for infra-solvmanifolds of type (R): $L$ is solvable of type (R), $N$ is of finite index in $E, W$ is a point and $\rho$ is the trivial action.

Write $\mathcal{H}^{f}(M)$ for the group of homeomorphisms of a model aspherical manifold $M$, associated to an $L$-set of data $(N, E, \rho, W)$, whose liftings to the universal cover $L \times W$ belong to $\mathcal{H}^{f}(L \times W)$. Elements of $\mathcal{H}^{f}(M)$ are called the fiber preserving homeomorphisms of $M$. Such liftings automatically belong to the normaliser of $\pi_{1}(M)=E$ in $\mathcal{H}^{f}(L \times W)$. A periodic map of the model aspherical manifold $M$ is a (non-trivial) element of finite order in $\mathcal{H}^{f}(M)$. In fact, $\mathcal{H}^{f}(M)$ can also be seen as the group of homeomorphisms of $M$ arising from conjugation in $N^{f}=$ $N_{\mathcal{H}^{f}(L \times W)}(E)$. We denote the corresponding epimorphism, with kernel isomorphic to $E$, by $q: N^{f} \rightarrow \mathcal{H}^{f}(M)$. This induces a natural homomorphism $\Phi: \mathcal{H}^{f}(M) \rightarrow$ $\operatorname{Out}(E)$ as follows: conjugating $E\left(\subset \mathcal{H}^{f}(L \times W)\right)$ with a lifting of $f \in \mathcal{H}^{f}(M)$ in $N^{f}$ induces an $E$-automorphism $\tilde{\Phi}(f)$ which represents $\Phi(f) \in \operatorname{Out}(E)$. 


\section{RIGID MODEL ASPHERICAL MANIFOLDS \\ ARISING FROM A SPLITTING AND FAITHFUL SET OF DATA}

Our interest goes to model aspherical manifolds $M$ without periodic maps, or equivalently, admitting no non-trivial finite groups of fiber preserving homeomorphisms acting effectively on $M$. In fact, we have the following sufficient condition:

Theorem 3.1. If $M$ is an aspherical manifold such that $\pi_{1}(M)$ is centerless and $\operatorname{Out}\left(\pi_{1}(M)\right)$ is torsion-free, then there are no effective actions on $M$ by a nontrivial finite group of homeomorphisms.

This result follows from a theorem of A. Borel ([CR71-1, Thm. 3.2]) stating that

Theorem 3.2. The abstract kernel $F \rightarrow \operatorname{Out}\left(\pi_{1}(M)\right)$, associated to a finite group $F$ acting effectively on an aspherical manifold $M$ with centerless fundamental group, is injective.

This was the starting point for F. Raymond and J.L. Tollefson ([RT76]) to construct examples of orientable aspherical 3-manifolds with no periodic maps. Taking off from the same theorem, in [CR71-2], P.E. Conner, F. Raymond and P.J. Weinberger also constructed aspherical manifolds (of dimensions 7, 11, 16, 22, 29 and 37) exhibiting this total lack of non-trivial finite, effective group actions. All these examples have solvable fundamental groups. Let us review the example of a 7 dimensional model aspherical solvmanifold without periodic maps.

Example 3.3. Let $N$ be the torsion-free, nilpotent group presented by

$$
N:=\langle a, b, c, d, e, f \mid[b, c]=d, \quad[c, a]=e, \quad[a, b]=f\rangle .
$$

Take the $N$-automorphism $\xi$ sending $a \mapsto b, b \mapsto c, c \mapsto a b^{-1}, d \mapsto d e, e \mapsto f$ and $f \mapsto d$. The semidirect product $E=N \rtimes_{\xi} \mathbb{Z}$ is a complete, torsion-free polycyclic group of Hirsch length 7 (see also [Rob80]). If $L$ denotes the $\mathrm{Mal}^{\prime}$ cev completion of $N$, which is a connected, simply connected nilpotent Lie group containing $N$ as a lattice and such that $(N, L)$ has $\operatorname{UAEP}([$ Mal51]), $W=\mathbb{R}$ and $\mathbb{Z}$ acts on $\mathbb{R}$ as translations (via $\rho$ ), then $(N, E, \rho, W)$ is an $L$-set of data determining a 7 dimensional model aspherical (solv)manifold with no periodic maps (because of Theorem 3.1).

Remark that the $\mathbb{Z}$-automorphism $\theta$ sending $z \in \mathbb{Z} \mapsto-z$ and the $\mathbb{R}$-homeomorphism $h$ sending $r \in \mathbb{R} \mapsto-r$ satisfy $\rho \circ \theta=\mu(h) \circ \rho$.

The set of eigenvalues of $\xi$, as an $L$-automorphism, are exactly the eigenvalues of the matrices $A=\left(\begin{array}{ccc}0 & 0 & 1 \\ 1 & 0 & -1 \\ 0 & 1 & 0\end{array}\right)$ and $B=\left(\begin{array}{lll}1 & 0 & 1 \\ 1 & 0 & 0 \\ 0 & 1 & 0\end{array}\right)$ ([Dek97]). These (rounded to six decimal places) are given by $0.682328,1.46557,-0.341164 \pm 1.16154 \mathrm{i}$ and $-0.232786 \pm 0.792552 \mathrm{i}$. Obviously, these eigenvalues are not of absolute value one and hence are not roots of unity. Therefore, as all eigenvalues of an inner automorphism of a simply connected, connected, nilpotent Lie group are one ([Par69, Lemma 2]), the abstract kernel $\psi: \mathbb{Z} \rightarrow \operatorname{Out}(L)$, induced by $1 \rightarrow N \rightarrow N \rtimes_{\xi} \mathbb{Z} \rightarrow$ $\mathbb{Z} \rightarrow 1$, is injective.

Inspired by this example, we define the following:

Definition 3.4. A model aspherical manifold $M$, associated to an $L$-set of data $(N, E, \rho, W)$, is rigid if and only if the action $\rho$ of $F=E / N$ on $W$ is rigid. That 
is, for each $\theta \in \operatorname{Aut}(F)$, there exists a homeomorphism $h \in \mathcal{H}(W)$ such that $\mu(h) \circ \rho=\rho \circ \theta$.

Definition 3.5. An $L$-set of data $(N, E, \rho, W)$ is faithful if and only if the abstract kernel $\psi: F \rightarrow \operatorname{Out}(L)$, induced by $1 \rightarrow N \rightarrow E \rightarrow F \rightarrow 1$ and the UAEP of $(N, L)$, is injective.

The following observations are rather elementary but nevertheless interesting:

Lemma 3.6. If $(N, E, \rho, W)$ is a faithful L-set of data, then the abstract kernel $F \rightarrow \operatorname{Out}(N)$, realising $1 \rightarrow N \rightarrow E \rightarrow F \rightarrow 1$, is also injective. If moreover $Z(N)$ is torsion-free, then $(N, E, \rho, W)$ is an admissible L-set of data.

Proof. This follows easily from Lemma 1.2.

Of crucial importance, however, is

Lemma 3.7. If $Z(N)$ is torsion-free and $(N, E, \rho, W)$ is a faithful $L$-set of data, then $N=E \cap L$.

Proof. Let $\Psi$ be a Seifert construction for the faithful $L$-set of data $(N, E, \rho, W)$. Write $\psi: F \rightarrow \operatorname{Out}(L)$ for the induced (injective) abstract kernel. Since $Z(N)$ is torsion-free, $\Psi$ is injective (Lemmas 1.3 and 3.6). Consider $e=(n, x) \in E(n \in N$, $x \in F)$ as an element $(\lambda, g, h) \in \Psi(E)$ and assume $e \in l(L)$. Then $g \in \operatorname{Inn}(L)$ and hence $\psi(x)=1$. Because of the injectivity of $\psi$, it follows that $e \in N$.

For this subclass of model aspherical manifolds $M$ (rigid and arising from a faithful set of data), we will investigate when $M$ admits no effective actions of any non-trivial finite group, or equivalently, when $M$ exhibits no periodic maps. We take off from the following result:

Proposition 3.8. If $M$ is a rigid model aspherical manifold arising from a faithful L-set of data $(N, E, \rho, W)$, then

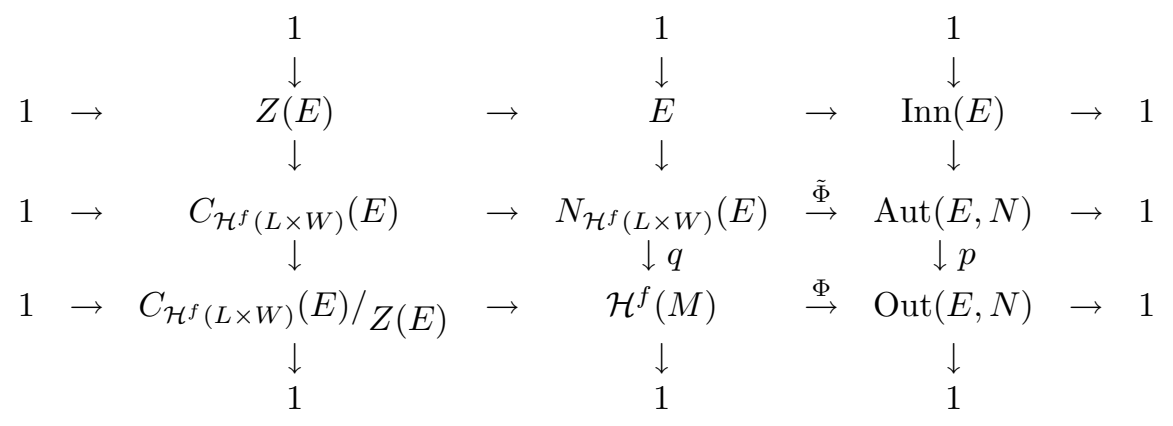

is a commutative diagram of exact rows and columns.

Proof. We already remarked that the kernel of the epimorphism $q: N_{\mathcal{H}^{f}(L \times W)}(E)=$ $N^{f} \rightarrow \mathcal{H}^{f}(M)$ is isomorphic to $E$, which yields the middle column.

Each element $(\lambda, g, h)$ of $N^{f}$ induces, via conjugation in $E$, an automorphism in $\operatorname{Aut}(E, N)$ since, for each $n \in N, \mu(\lambda, g, h)(n, \mu(n), 1)=(g(n), \mu(g(n)), 1)$ and $g(n) \in E \cap L=N$ because of Lemma $3.7(Z(N)$ is torsion-free as subgroup of $E)$. Write $\tilde{\Phi}: N^{f} \rightarrow \operatorname{Aut}(E, N)$ for this group homomorphism. Obviously, the kernel of $\tilde{\Phi}$ equals $C_{\mathcal{H}^{f}(L \times W)}(E)$. We claim that $\tilde{\Phi}$ is onto. 
Each $\sigma \in \operatorname{Aut}(E, N)$ induces an $F$-automorphism $\bar{\sigma}$. Since the action of $F$ on $W$ is rigid, there exists a homeomorphism $h \in \mathcal{H}(W)$ such that $\mu(h) \circ \rho=\rho \circ \bar{\sigma}$. If $\Psi$ is a Seifert construction for the set of data $(N, E, \rho, W)$, then, because of the rigidity of Seifert constructions, $\Psi \circ \sigma=\mu(\hat{h}) \circ \Psi$, for some $\hat{h} \in \mathcal{H}^{f}(L \times W)$. Hence $\tilde{\Phi}(\hat{h})=\sigma$.

Remark 3.9. The following example shows that the above theorem does not hold for a general (not necessarily faithful) set of data, as the image of an element of $N_{\mathcal{H}^{f}(L \times W)}(E)$ under $\tilde{\Phi}$ is not necessarily in $\operatorname{Aut}(E, N)$. Hence, it also indicates that Theorem 3.3 of [KLR83] should be formulated more carefully.

Example 3.10. Consider the short exact sequence $1 \rightarrow N=2 \mathbb{Z} \times \mathbb{Z} \cong \mathbb{Z}^{2} \rightarrow E=$ $\mathbb{Z}^{2} \rightarrow \mathbb{Z}_{2} \rightarrow 1$, which induces a trivial abstract kernel $\psi: \mathbb{Z}_{2} \rightarrow$ Aut $\left(\mathbb{R}^{2}\right)$. Observe that $E \cap \mathbb{R}^{2}=E \neq N$. Let $W$ be a point and $\rho$ the trivial action. Then $\Psi: E \rightarrow$ $\operatorname{Aff}\left(\mathbb{R}^{2}\right)=\mathbb{R}^{2} \rtimes \operatorname{Aut}\left(\mathbb{R}^{2}\right)$, which sends $\left(z_{1}, z_{2}\right) \in \mathbb{Z}^{2} \mapsto\left(\left(z_{1}, z_{2}\right),\left(\begin{array}{ll}1 & 0 \\ 0 & 1\end{array}\right)\right)$ is a Seifert construction for the $\mathbb{R}^{2}$-set of data $(N, E, \rho, W)$. However, the affine transformation $\left((0,0),\left(\begin{array}{ll}0 & 1 \\ 1 & 0\end{array}\right)\right)$ normalises $E$ but does not restrict to an automorphism of $N$, as it sends $\left(2 z_{1}, z_{2}\right) \in N \mapsto\left(z_{2}, 2 z_{1}\right)$.

An interesting situation arises when the short exact sequence $1 \rightarrow N \rightarrow E \rightarrow$ $F \rightarrow 1$, associated to an $L$-set of data $(N, E, \rho, W)$, can be embedded into a splitting extension of $L$ by $F$.

Definition 3.11. An $L$-set of data $(N, E, \rho, W)$ is $\varphi$-splitting if and only if there is a splitting extension $1 \rightarrow L \rightarrow \mathcal{E} \rightarrow F=E / N \rightarrow 1$ inducing an action $\varphi: F \rightarrow$ $\operatorname{Aut}(L)$ of $F$ on $L$, such that

$$
\begin{aligned}
& \begin{array}{lllllll}
N & \rightarrow & E & \rightarrow & F & \rightarrow & 1 \\
& & \downarrow & & & &
\end{array} \\
& 1 \rightarrow L \rightarrow \mathcal{E}=L \rtimes_{\varphi} F \rightarrow F \rightarrow 1
\end{aligned}
$$

is commutative.

In this case, there is a natural action of $F$ on $\mathcal{M}\left(W, L^{*}\right)$.

Lemma 3.12. If $(N, E, \rho, W)$ is a $\varphi$-splitting $L$-set of data, then $F=E / N$ acts on $\mathcal{M}\left(W, L^{*}\right)$ as follows: for $x \in F$ and $\lambda \in \mathcal{M}\left(W, L^{*}\right),{ }^{x} \lambda=\varphi(x) \circ \lambda \circ \rho(x)^{-1}$.

Of crucial importance here is that

Lemma 3.13. Let $(N, E, \rho, W)$ be a $\varphi$-splitting L-set of data. Write $\psi: F \rightarrow$ $\operatorname{Out}(N)$ for the abstract kernel induced by $1 \rightarrow N \rightarrow E \rightarrow F \rightarrow 1$. Then, for any lift $\varphi^{\prime}: F \rightarrow \operatorname{Aut}(N)$ of $\psi$, there is a commutative diagram (of maps)

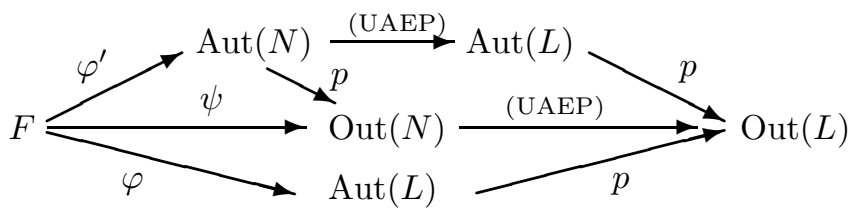

Proof. Fix a section $s: F \rightarrow E$ of the short exact sequence $1 \rightarrow N \rightarrow E \rightarrow F \rightarrow 1$. This induces, via conjugation in $E$, a lift $\varphi^{\prime}: F \rightarrow \operatorname{Aut}(N)$ of the abstract kernel 
$\psi: F \rightarrow \operatorname{Out}(N)$. Since any other lift of $\psi$ differs from $\varphi^{\prime}$ by inner automorphisms of $N$, it is enough to prove the claim for this specific $\varphi^{\prime}$.

Consider $x \in F$ and let $e=s(x) \in E$. Seen as an element of $L \rtimes_{\varphi} F$, we can write $e=\left(l_{x}, x\right)$, for some $l_{x} \in L$. We verify that, for each $n \in N$ :

$$
\left(\varphi^{\prime}(x)(n), 1\right)=\left(l_{x}, x\right)(n, 1)\left(\varphi(x)^{-1}\left(l_{x}^{-1}\right), x^{-1}\right)=\left(l_{x} \cdot \varphi(x)(n) \cdot l_{x}^{-1}, 1\right),
$$

showing that $\varphi^{\prime}(x)=\mu\left(l_{x}\right) \circ \varphi(x)$ on $N$. That both maps coincide on the whole of $L$ follows from the UAEP of $(N, L)$.

This implies

Corollary 3.14. If $(N, E, \rho, W)$ is a $\varphi$-splitting and faithful $L$-set of data, then $\varphi: F=E / N \rightarrow \operatorname{Aut}(L)$ is an effective action of $F$ on $L$.

Now, for a suitable $L$-set of data $(N, E, \rho, W)$, the centraliser of $E$ in $\mathcal{H}^{f}(L \times W)$ can be described as follows:

Proposition 3.15. If $(N, E, \rho, W)$ is an admissible, splitting L-set of data and $F=E / N$, then

$$
C_{\mathcal{H}^{f}(L \times W)}(E)=\mathcal{M}\left(W, L^{*}\right)^{F} \rtimes C_{\mathcal{H}(W)}(\rho(F)),
$$

where $\mathcal{M}\left(W, L^{*}\right)^{F}$ denotes the subgroup of elements of $\mathcal{M}\left(W, L^{*}\right)$ fixed under the action of $F$.

Proof. Since $(N, E, \rho, W)$ is a splitting $L$-set of data, there is an action $\varphi: F \rightarrow$ $\operatorname{Aut}(L)$ such that $E$ can be embedded in $L \rtimes_{\varphi} F$. Take a section $s: F \rightarrow E$ of the short exact sequence $1 \rightarrow N \rightarrow E \rightarrow F \rightarrow 1$. Seen as an element of $L \rtimes_{\varphi} F$, $s(x)=\left(l_{x}, x\right)\left(l_{x} \in L, x \in F\right)$. This section also induces a 2-cocycle $c: F \times F \rightarrow N$ satisfying, for each $x, y \in F$,

$$
c(x, y) \cdot l_{x y}=l_{x} \cdot \varphi(x)\left(l_{y}\right) .
$$

Write $\Psi: E \rightarrow \mathcal{H}^{f}(L \times W)$ for the map sending $(n, x) \in E \mapsto\left(n \cdot l_{x}, \mu\left(n \cdot l_{x}\right) \varphi(x), \rho(x)\right)$. We claim that $\Psi$ is a homomorphism. Obviously, since $\Psi(n, x)=\Psi(n, 1) \Psi(1, x)$ (for all $n \in N$ and $x \in F$ ), it is sufficient that $\Psi((1, x)(1, y))=\Psi(1, x) \Psi(1, y)$, for any $x, y \in F$ :

$$
\begin{aligned}
\Psi((1, x)(1, y)) & =\Psi(c(x, y), x y)=\left(c(x, y) \cdot l_{x y}, \mu\left(c(x, y) \cdot l_{x y}\right) \varphi(x y), \rho(x y)\right) \\
& =\left(l_{x} \cdot \varphi(x)\left(l_{y}\right), \mu\left(l_{x} \cdot \varphi(x)\left(l_{y}\right)\right) \varphi(x) \varphi(y), \rho(x) \rho(y)\right) \\
& =\left(l_{x}, \mu\left(l_{x}\right) \varphi(x), \rho(x)\right)\left(l_{y}, \mu\left(l_{y}\right) \varphi(y), \rho(y)\right)=\Psi(1, x) \Psi(1, y) .
\end{aligned}
$$

Moreover, if $\psi: F \rightarrow \operatorname{Out}(L)$ denotes the homomorphism induced by the extension $1 \rightarrow N \rightarrow E \rightarrow F \rightarrow 1$ and the UAEP of $(N, L)$, then, because of Lemma 3.13, the following diagram is commutative:

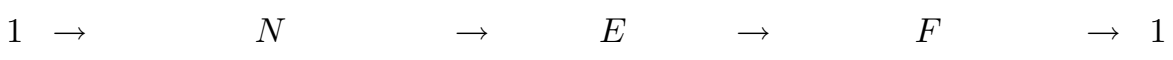

$$
\begin{aligned}
& 1 \rightarrow \mathcal{M}\left(W, L^{*}\right) \rtimes \operatorname{Inn}(L) \rightarrow \mathcal{H}^{f}(L \times W) \rightarrow \operatorname{Out}(L) \times \mathcal{H}(W) \rightarrow 1
\end{aligned}
$$

Hence $\Psi$ is a Seifert construction for the $L$-set of data $(N, E, \rho, W)$.

Now, concentrate on the centraliser of $\Psi(E) \cong E$ ( $\Psi$ is injective, as the set of data is admissible) in $\mathcal{H}^{f}(L \times W)$. Take $(\lambda, g, h) \in \mathcal{H}^{f}(L \times W)$ and $n \in N$. Then

$$
(\lambda, g, h) \Psi(n, 1)=(\lambda, g, h)(n, \mu(n), 1)=(g(n) \cdot \lambda, g \mu(n), h)
$$


and

$$
\Psi(n, 1)(\lambda, g, h)=(n, \mu(n), 1)(\lambda, g, h)=(n \cdot \lambda, \mu(n) g, h) .
$$

Hence, a necessary condition for $(\lambda, g, h)$ to belong to $C_{\mathcal{H}^{f}(L \times W)} \Psi(E)$ is that $g$ is the identity on $N$ and hence on $L$ (via UAEP). Moreover, for all $x \in F$,

$$
(\lambda, 1, h) \Psi(1, x)=(\lambda, 1, h)\left(l_{x}, \mu\left(l_{x}\right) \varphi(x), \rho(x)\right)=\left(l_{x} \cdot \lambda, \mu\left(l_{x}\right) \varphi(x), h \rho(x)\right)
$$

and

$$
\begin{aligned}
\Psi(1, x)(\lambda, 1, h) & =\left(l_{x}, \mu\left(l_{x}\right) \varphi(x), \rho(x)\right)(\lambda, 1, h) \\
& =\left(l_{x} \cdot\left(\varphi(x) \circ \lambda \circ \rho(x)^{-1}\right), \mu\left(l_{x}\right) \varphi(x), \rho(x) h\right) .
\end{aligned}
$$

We deduce that $(\lambda, 1, h) \in C_{\mathcal{H}^{f}(L \times W)} \Psi(E)$ if and only if $h \in C_{\mathcal{H}(W)}(\rho(F))$ and, for all $x \in F, \varphi(x) \circ \lambda=\lambda \circ \rho(x)$, or equivalently $\lambda \in \mathcal{M}\left(W, L^{*}\right)^{F}$.

As a consequence of Propositions 3.8 and 3.15, we have

Proposition 3.16. If $M$ is a rigid model aspherical manifold arising from a splitting and faithful L-set of data $(N, E, \rho, W)$ and $F=E / N$, then

$$
1 \rightarrow\left(\mathcal{M}\left(W, L^{*}\right)^{F} \rtimes C_{\mathcal{H}(W)}(\rho(F))\right) / Z(E) \rightarrow \mathcal{H}^{f}(M) \stackrel{\Phi}{\rightarrow} \operatorname{Out}(E, N) \rightarrow 1
$$

is exact.

Hence, because of Borel's Theorem (Theorem 3.2), it follows that

Corollary 3.17. Assume $M$ is a rigid model aspherical manifold associated to a splitting and faithful $L$-set of data $(N, E, \rho, W)$. Then, if $E$ is centerless, the semidirect product $\mathcal{M}\left(W, L^{*}\right)^{F} \rtimes C_{\mathcal{H}(W)}(\rho(F))$ (where $\left.F=E /{ }_{N}\right)$ is torsion-free.

Problem 3.18. Under the conditions of Corollary 3.17, $L$ is torsion-free (as $L$ is connected and contractible) and hence $\mathcal{M}\left(W, L^{*}\right)^{F}$ is without torsion. We invite the reader to formulate a straightforward argument (that is, without referring to Borel's theorem) to show that $C_{\mathcal{H}(W)}(\rho(F))$ is torsion-free.

Conversely, we have

Lemma 3.19. Let $(N, E, \rho, W)$ be an admissible and splitting L-set of data such that $1 \rightarrow N \rightarrow E \rightarrow F \rightarrow 1$ realises an injective abstract kernel. Assume $Z(L)$ is connected and torsion-free. If $\left(\mathcal{M}\left(W, L^{*}\right)^{F} \rtimes C_{\mathcal{H}(W)}(\rho(F))\right) / Z(E)$ is torsion-free, then $E$ is centerless.

Proof. Assume the center of $E$ is not trivial. Since $1 \rightarrow N \rightarrow E \rightarrow F \rightarrow 1$ induces an injective abstract kernel, $Z(N)^{F}=Z(E)$ (Lemma 1.1). Because $Z(L)$ is an abelian, connected and torsion-free Lie group, $Z(L)$ is isomorphic to the vector space $\mathbb{R}^{k}$, for some $k \in \mathbb{N}$. Moreover, $Z(N)^{F}$ is a (non-trivial) discrete subgroup of $Z(L)^{F}$ since $N$ is a discrete subgroup of $L$ and $N \cap Z(L)=Z(N)$ (because of the UAEP of $(N, L))$. Hence $Z(L)^{F} / Z(E)$ contains a non-trivial torus and there is torsion in $\left(\mathcal{M}\left(W, L^{*}\right)^{F} \rtimes C_{\mathcal{H}(W)}(\rho(F))\right) / Z(E)$.

Now we are ready to formulate, for a subclass of model aspherical manifolds $M$ (arising from "nice" sets of data), an equivalent algebraic condition for there to be no effective actions of non-trivial finite groups on $M$ (or $M$ is without periodic maps). Remark that the first statement is Theorem 3.1, but now for model aspherical manifolds. 
Theorem 3.20. Let $M$ be a rigid model aspherical manifold arising from a splitting and faithful $L$-set of data $(N, E, \rho, W)$. Then

1. If $E$ is centerless and $\operatorname{Out}(E, N)$ is torsion-free, then $\mathcal{H}^{f}(M)$ is torsion-free.

2. If every finite extension of $\mathcal{M}\left(W, L^{*}\right)^{F} \rtimes C_{\mathcal{H}(W)}(\rho(F))$ splits, $Z(L)$ is connected and $\mathcal{H}^{f}(M)$ is torsion-free, then $E$ is centerless and $\operatorname{Out}(E, N)$ is torsion-free.

3. If $Z(L)$ is connected, then $\mathcal{H}^{f}(M)$ is trivial if and only if both $\operatorname{Out}(E, N)$ and $\mathcal{M}\left(W, L^{*}\right)^{F} \rtimes C_{\mathcal{H}(W)}(\rho(F))$ are trivial.

Proof. The first statement follows easily from Proposition 3.16 and Corollary 3.17.

If $\mathcal{H}^{f}(M)$ is torsion-free, it follows from Lemma 3.19 (the abstract kernel induced by $1 \rightarrow N \rightarrow E \rightarrow F \rightarrow 1$ is injective by Lemma 3.6) that $E$ is centerless (note that, at this point, we have already proved the final claim). Now, assume there is an element $f \in \mathcal{H}^{f}(M)$ such that $\Phi(f)^{k}=1$, for some $k \in \mathbb{N}_{0}$. Take the finite subgroup $G$ of $\operatorname{Out}(E, N)$ generated by $\Phi(f)$ and consider the short exact sequence $1 \rightarrow \mathcal{M}\left(W, L^{*}\right)^{F} \rtimes C_{\mathcal{H}(W)}(\rho(F)) \rightarrow \widetilde{A} \stackrel{\Phi}{\rightarrow} G \rightarrow 1$, where $\widetilde{A}$ is the preimage of $G$ under $\Phi$. It is given that this extension splits and hence $\mathcal{H}^{f}(M)$ has torsion.

\section{Centerless extensions Realising an injective Abstract Kernel and THEIR AUTOMORPHISMS}

To apply Theorem 3.20 , it is necessary to be able to decide when a group $E$, fitting into an extension $1 \rightarrow N \rightarrow E \rightarrow F \rightarrow 1$ realising an injective abstract kernel $F \rightarrow \operatorname{Out}(N)$, is centerless, and when moreover $\operatorname{Out}(E, N)$ is torsionfree. In [IM94], commutative diagrams were developed to describe $\operatorname{Aut}(E, N)$ and $\operatorname{Out}(E, N)$. We briefly recall the theoretical set-up to construct these fundamental automorphism diagrams, and refer to [IM94] for more details.

Assume $1 \rightarrow N \rightarrow E \rightarrow F \rightarrow 1$ is a group extension compatible with an injective abstract kernel $\psi: F \rightarrow \operatorname{Out}(N)$ and determining a cohomology class $a \in H_{\psi}^{2}(F, N)$ (where $H_{\psi}^{2}(F, N)$ denotes the set of 2-cohomology classes compatible with $\psi$ ). It is well known that $H_{\psi}^{2}(F, N)$ is in one-to-one correspondence with $H_{\psi}^{2}(F, Z(N))$ if there is an extension realising $\psi$. We define a subgroup $\mathcal{M}_{\psi}$ of $\operatorname{Aut}(N)$ by $\mathcal{M}_{\psi}=p^{-1}\left(N_{\operatorname{Out}}(N) \psi(F)\right)$, the preimage in $\operatorname{Aut}(N)$ of the normaliser of $\psi(F)$ in $\operatorname{Out}(N)$. Write ${ }^{*}: \mathcal{M}_{\psi} \rightarrow \operatorname{Aut}(F)$ for the group homomorphism defined by $\nu^{*}=\psi^{-1} \circ \mu(p(\nu)) \circ \psi$, for each $\nu \in \mathcal{M}_{\psi}$. Write $\mathcal{M}_{\psi, a}$ for the stabiliser of $a$ under the corresponding action of $\mathcal{M}_{\psi}$ on $H_{\psi}^{2}(F, N)$. Each automorphism $\sigma$ in $\operatorname{Aut}(E, N)$ restricts to an automorphism of $N$ via a homomorphism $A: \operatorname{Aut}(E, N) \rightarrow \operatorname{Aut}(N)$. This induces short exact sequences

$$
1 \rightarrow Z_{\psi}^{1}(F, Z(N)) \rightarrow \operatorname{Aut}(E, N) \stackrel{A}{\rightarrow} \mathcal{M}_{\psi, a} \rightarrow 1
$$

and

$$
1 \rightarrow H_{\psi}^{1}(F, Z(N)) \rightarrow \operatorname{Out}(E, N) \rightarrow Q \rightarrow 1,
$$

where the quotient $Q$ equals

$$
Q=\overline{\mathcal{M}_{\psi, a}} / F \quad \text { and } \quad \overline{\mathcal{M}_{\psi, a}}=\mathcal{M}_{\psi, a} / \operatorname{Inn}(N) .
$$

In the following proposition, we characterise algebraically centerless groups $E$, fitting into an extension with kernel $N$ and realising an injective abstract kernel, such that $\operatorname{Out}(E, N)$ is trivial. 
Proposition 4.1. Let $1 \rightarrow N \rightarrow E \rightarrow F \rightarrow 1$ be an extension realising an injective abstract kernel $\psi: F \rightarrow \operatorname{Out}(N)$ and determining a cohomology class a $\in H_{\psi}^{2}(F, N)$. Then

1. If $F$ is finite, $E$ is centerless and $\operatorname{Out}(E, N)$ is torsion-free, then $H_{\psi}^{0}(F, Z(N))$ and $H_{\psi}^{1}(F, Z(N))$ are trivial.

2. $Z(E)=\{1\}=\operatorname{Out}(E, N)$ if and only if $H_{\psi}^{0}(F, Z(N))=\{1\}=H_{\psi}^{1}(F, Z(N))$ and $\overline{\mathcal{M}_{\psi, a}}=\psi(F)$.

3. If $F$ is self-normalising in $\operatorname{Out}(N)$, then $\overline{\mathcal{M}_{\psi, a}}=\psi(F)$ (and $\left.\mathcal{M}_{\psi, a}=\mathcal{M}_{\psi}\right)$.

4. $F$ is self-normalising in $\operatorname{Out}(N)$ if and only if $C_{\operatorname{Out}(N)} \psi(F)=Z(F)$ and $\mathcal{M}_{\psi}^{*}=\operatorname{Inn}(F)$.

5. $E$ is centerless, $\operatorname{Out}(E, N)$ is trivial and $\mathcal{M}_{\psi, a}=\mathcal{M}_{\psi}$ if and only if $F$ is self-normalising in $\operatorname{Out}(N)$ and $H_{\psi}^{0}(F, Z(N))=\{1\}=H_{\psi}^{1}(F, Z(N))$.

Proof. Because of Lemma 1.1, $Z(E)=Z(N)^{F} \cong H_{\psi}^{0}(F, Z(N))$, and the first two claims follow easily from (1) and (2).

Assume $F$ is self-normalising in $\operatorname{Out}(N)$. Then, because of the definition of $\mathcal{M}_{\psi}$, it follows that $\overline{\mathcal{M}_{\psi}}=\overline{\mathcal{M}_{\psi, a}}=\psi(F)$ and $\mathcal{M}_{\psi, a}=\mathcal{M}_{\psi}$. Moreover, for each $\nu \in \mathcal{M}_{\psi}$ we have $p(\nu)=\psi\left(x_{\nu}\right)$ for some $x_{\nu} \in F$, and hence $\nu^{*}=\mu\left(x_{\nu}\right)$ or $\mathcal{M}_{\psi}^{*}=\operatorname{Inn}(F)$.

Conversely, if $\mathcal{M}_{\psi}^{*}=\operatorname{Inn}(F)$, then, for each $\nu \in \mathcal{M}_{\psi}$, there is an element $x_{\nu} \in F$ such that $p(\nu) \psi\left(x_{\nu}\right) \in C_{\mathrm{Out}}(N) \psi(F)$. Since this centraliser is in $\psi(F), p(\nu)$ belongs to $\psi(F)$ and consequently $F$ is self-normalising in $\operatorname{Out}(N)$.

Let us remark the following about the situation $\mathcal{M}_{\psi, a}=\mathcal{M}_{\psi}$.

Remark 4.2. If $N$ is abelian and $1 \rightarrow N \rightarrow E \rightarrow F \rightarrow 1$ splits, then $\mathcal{M}_{\psi, a}=\mathcal{M}_{\psi}$. This does not hold for general $N$ (we refer to [IM94], where this is illustrated). Of course, if $H_{\psi}^{2}(F, Z(N))=\{1\}$ (or $H_{\psi}^{2}(F, N)=\{a\}$ ), then $\mathcal{M}_{\psi, a}$ coincides with $\mathcal{M}_{\psi}$. However, $H_{\psi}^{2}(F, Z(N))$ being trivial does not imply that $1 \rightarrow N \rightarrow E \rightarrow F \rightarrow 1$ splits. For example, consider the group $E$ presented as:

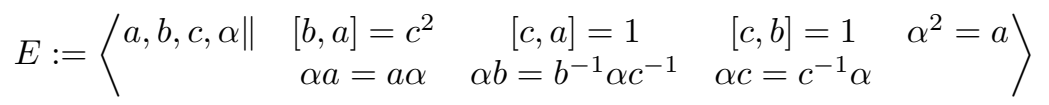

and fitting into the extension $1 \rightarrow N \rightarrow E \rightarrow \mathbb{Z}_{2} \rightarrow 1$, where

$$
N=<a, b, c \|[b, a]=c^{2}[c, a]=1[c, b]=1>.
$$

Obviously, the center of $N$ is the subgroup generated by $c$, and since $\mathbb{Z}_{2}$ acts nontrivially on it, $H^{2}(F, Z(N))=\{1\}$, although $E$ is clearly torsion-free.

Let us now concentrate on the crucial necessary condition that $H_{\psi}^{0}(F, Z(N))$ and $H_{\psi}^{1}(F, Z(N))$ both vanish. In this perspective, the following is interesting:

Lemma 4.3 ([Rob80]). Let $F$ be a nilpotent group or an extension of a cyclic group by a cyclic p-group. Assume $A$ is a finitely generated free abelian $F$-module. If $H^{0}(F, A)=\{1\}=H^{1}(F, A)$, then $H^{2}(F, A)=\{1\}$.

Because of proposition 4.1.5 and remark 4.2, this implies that

Proposition 4.4 (cf. [Rob80, Lemma 4]). Let $1 \rightarrow N \rightarrow E \rightarrow F \rightarrow 1$ be an extension realising an injective abstract kernel $\psi$ and such that $Z(N)$ is finitely generated and torsion-free. Assume $F$ is nilpotent or an extension of a cyclic group by a cyclic p-group. Then $E$ is centerless and $\operatorname{Out}(E, N)$ is trivial if and only if $F$ is self-normalising in $\operatorname{Out}(N)$ and $H_{\psi}^{0}(F, Z(N))=\{1\}=H_{\psi}^{1}(F, Z(N))$. 
The following lemma implies that there are no centerless extensions $E$ (realising an injective abstract kernel), with kernel $N$ (such that $Z(N)$ is finitely generated and torsion-free) and a finite $p$-group as end group, $\operatorname{such}$ that $\operatorname{Out}(E, N)$ is torsionfree (because of Proposition 4.1.1).

Lemma 4.5. If $F$ is a finite $p$-group and $\mathbb{Z}^{k}$ an $F$-module, then $H^{0}\left(F, \mathbb{Z}^{k}\right)=\{1\}$ implies that $H^{1}\left(F, \mathbb{Z}^{k}\right) \neq\{1\}$.

Proof. By assumption we have that $\left(\mathbb{Q}^{k} / \mathbb{Z}^{k}\right)^{F} \cong H^{0}\left(F, \mathbb{Q}^{k} / \mathbb{Z}^{k}\right) \cong H^{1}\left(F, \mathbb{Z}^{k}\right)$. The

$F$-module structure on $\mathbb{Q}^{k} / \mathbb{Z}^{k}$ restricts to an action of $F$ on $\mathbb{Z}_{p}{ }^{k}=\mathbb{Z}^{k} / p \mathbb{Z}^{k}$. The orders $c_{i}$ of the orbits of this action are powers of $p$. At least one $c_{i}$ is 1 (namely the orbit of the identity). However, $\sum c_{i}=p^{k}$, which implies that at least one other $c_{i}$ is 1 or there is a fixed point.

Remark 4.6. Unfortunately, this lemma does not hold for a wider class of finite groups. Indeed, even for cyclic groups this result is, in general, not true anymore. For example, let $F$ be the cyclic subgroup of $\operatorname{Gl}(2, \mathbb{Z})$ generated by $\left(\begin{array}{cc}1 & -1 \\ 1 & 0\end{array}\right)$ (or $\left.F \cong \mathbb{Z}_{6}\right)$. The reader can easily verify that $H^{0}\left(F, \mathbb{Z}^{2}\right)$ and $H^{1}\left(F, \mathbb{Z}^{2}\right)$ both vanish.

\section{Model ASPherical MANIFOLds With A POINT AS BASE}

An interesting situation where rigid model aspherical manifolds $M$ arise quite naturally is when we consider $L$-sets of data $(N, E, \rho, W)$, where $W$ is a point $(W=\{\cdot\}), \rho$ is the trivial action and $N$ is of finite index in $E$.

Then the group of fiber preserving homeomorphisms of $L$ compatible with left translations is $L^{*} \rtimes \operatorname{Aut}(L)$. Here $L^{*}$ is the group with underlying set $L$ but the multiplication $*$ is given by reversing the order of the elements. This group is clearly isomorphic to the classical affine group $\operatorname{Aff}(L)=L \rtimes \operatorname{Aut}(L)$ of the Lie group $L$ (via the isomorphism $L^{*} \rtimes \operatorname{Aut}(L) \rightarrow \operatorname{Aff}(L)$ sending $(l, g) \in L^{*} \rtimes \operatorname{Aut}(L) \mapsto$ $\left.\left(l, \mu\left(l^{-1}\right) g\right)\right)$. Write $\operatorname{Aff}(M)$ for the group of homeomorphisms of $M$ whose liftings to the universal cover $L$ belong to $\operatorname{Aff}(L)$.

As a consequence of Proposition 3.16 and Theorem 3.20, we have

Theorem 5.1. Assume $M$ is a model aspherical manifold arising from a splitting and faithful L-set of data $(N, E, \rho,\{\cdot\})$, where $F=E / N$ is finite and $\rho$ is the trivial action. Then

$$
1 \rightarrow L^{F} / Z(E) \rightarrow \operatorname{Aff}(M) \rightarrow \operatorname{Out}(E, N) \rightarrow 1
$$

is exact.

If $Z(L)$ is connected, then $\operatorname{Aff}(M)$ is trivial if and only if both $L^{F}$ and $\operatorname{Out}(E, N)$ are trivial. If moreover every finite extension of $L^{F}$ splits, then $\mathrm{Aff}(M)$ is torsionfree if and only if $E$ is centerless and $\operatorname{Out}(E, N)$ is torsion-free.

Let us now concentrate on the following rather well known classes of rigid model aspherical manifolds arising from a splitting and faithful set of data, and see if we can apply the theorem above.

5.1. Infra-solvmanifolds of type $(\mathbf{R})$. Let $L$ be a connected, simply connected solvable Lie group. Assume $L$ is of type $(\mathrm{R})$. That is, for each element $x$ of the Lie algebra of $L, \operatorname{ad}(x)$ has only real eigenvalues. It is well known that the center of $L$ is connected, and if $N$ is a lattice in $L,(N, L)$ has UAEP ([Gor71],[Gor73]). 
Assume $E$ is a torsion-free extension of a lattice $N$ in $L$ by a finite group $F$. Then $E$ can be embedded into $\operatorname{Aff}(L)$ via a Seifert construction (arising from the $L$-set of data $(N, E, \rho,\{\cdot\})$, where $\rho$ is trivial). The model aspherical manifold $M=E \backslash L$ arising from such a set of data is called an infra-solvmanifold $M$ of type (R).

If $E$ is a finite extension of a lattice $N$ in $L$, then $E \cap L$ is a lattice in $L$ and a characteristic subgroup of finite index in $E$ ([Lee95, Prop.4.2]). Hence, without loss of generality, we can assume that $N$ is the translation subgroup $E \cap L$ of $E$. Remark that $(N, E, \rho,\{\cdot\})$ is a splitting $L$-set of data since each extension of $L$ by a finite group splits ([Lee95, Lemma 2.1]). Moreover, for this particular set of data, also the converse of Lemma 3.7 holds:

Lemma 5.2. Let $L$ be a connected, simply connected solvable Lie group of type (R). Assume $E \cap L$ is a lattice in $L$ and $E$ is an admissible, finite extension of $E \cap L$. Then $(E \cap L, E, \rho,\{\cdot\})$ is a faithful L-set of data.

Proof. Since $1 \rightarrow N=E \cap L \rightarrow E \rightarrow F \rightarrow 1$ is an admissible extension, $E$ can be embedded in $\operatorname{Aff}(L)$ via a Seifert construction for the $L$-set of data $(E \cap L, E, \rho,\{\cdot\})$. Now take $\bar{x} \in F=E / N$, where $x=(g, \alpha) \in E(g \in L, \alpha \in \operatorname{Aut}(L))$. If $\psi: F \rightarrow$ $\operatorname{Out}(L)$ denotes the abstract kernel induced by $1 \rightarrow N \rightarrow E \rightarrow F \rightarrow 1$ and the UAEP of $(N, L)$, then $\psi(\bar{x})=\alpha \operatorname{Inn}(L)$. Assume $\psi(\bar{x})=1$. Then $\alpha$ is an inner automorphism of $L$ (and hence has only positive real eigenvalues ([Dek97])). On the other hand, since $F$ is finite, $\alpha$ is an automorphism of finite order (which has, if $\alpha$ is not the identity, at least one eigenvalue which is not a positive real number ([Dek97])). It follows that $\alpha$ must be the identity or $x=(g, 1) \in E \cap L=N$ and $\bar{x}=1$.

We conclude that each infra-solvmanifold $M$ of type (R) arises from a splitting and faithful $L$-set of data $(N, E, \rho,\{\cdot\})$, where $F=E / N$ is finite and $\rho$ is trivial. Moreover, since $L$ is a simply connected exponential Lie group, the Lie subgroup $L^{F}$ is again connected and simply connected. This guarantees that each finite extension of $L^{F}$ splits ([Lee95, Lemma 2.1]), and we conclude that

Theorem 5.3. There are no non-trivial finite groups of affine diffeomorphisms acting effectively on an infra-solvmanifold $M$ of type $(R)$ if and only if $\pi_{1}(M)$ is centerless and $\operatorname{Out}\left(\pi_{1}(M)\right)$ is torsion-free.

Remark 5.4. We should note that a straightforward and independent proof of this theorem follows from [Lee95]:

If $\pi_{1}(M)$ had a non-trivial center, $M$ would admit a non-trivial affine torus action. Hence, $\pi_{1}(M)$ must be centerless. If now $\mathbb{Z}_{p} \subseteq \operatorname{Out}\left(\pi_{1}(M)\right)$, for some $p$, then there always exists an (admissible) extension $1 \rightarrow \pi_{1}(M) \rightarrow E \rightarrow \mathbb{Z}_{p} \rightarrow 1$ realising this faithful abstract kernel. Therefore, $\mathbb{Z}_{p}$, as a subgroup of $\operatorname{Out}\left(\pi_{1}(M)\right)$, lifts to an effective affine action, and this contradicts the assumption.

5.2. Infra-nilmanifolds and flat Riemannian manifolds. Let $L$ be a connected and simply connected, nilpotent Lie group. Let $C$ be a maximal compact subgroup of $\operatorname{Aut}(L)$. An almost-crystallographic group (of $L$ ) is a discrete and uniform subgroup $E$ of $L \rtimes C \subset \operatorname{Aff}(L)$. The translation subgroup $N=E \cap L$ is a lattice in $L$ and is the unique normal subgroup of $E$ which is maximal nilpotent ([Aus60]). Moreover, in [Dek96], it was shown that $N$ is maximal nilpotent in $E$ if and only if the induced abstract kernel $F=E / N \rightarrow \operatorname{Out}(L)$ is injective. 
Hence, the finite quotient $F$, which is called the holonomy group, acts faithfully on $L$ (Corollary 3.14).

As an abstract group, a group $E$ is almost-crystallographic if and only if it contains a torsion-free, finitely generated nilpotent normal subgroup $N$ of finite index, which is maximal nilpotent in $E$ ([LR84]). Then $N$ is the Fitting subgroup of $E$ ([Seg83]). In this case the Lie group $L$ is the $\mathrm{Mal}^{\prime} \mathrm{cev}$ completion of $N$. The corresponding extension $1 \rightarrow N \rightarrow E \rightarrow F \rightarrow 1$ is called essential. A basic fact concerning essential extensions is that they induce an injective abstract kernel ([DIM93]). Also observe that $N$ is characteristic in $E$.

If $E$ is torsion-free, then $E$ is called an almost-Bieberbach group and the corresponding compact orbit space $M=E \backslash L$ is an infra-nilmanifold. Therefore, almost-Bieberbach groups are exactly the fundamental groups of infra-nilmanifolds. Clearly, this set-up is a straightforward generalisation of the classical crystallographic (resp. Bieberbach) groups and flat Riemannian manifolds (i.e. the situation $L=\mathbb{R}^{n}$ ) and, on the other hand, infra-nilmanifolds are infra-solvmanifolds of type (R). Because of Theorem 5.1, we have

Theorem 5.5. An infra-nilmanifold $M$, with universal cover $L$ and holonomy group $F$, admits no non-trivial finite, effective actions via affine diffeomorphisms if and only if $\pi_{1}(M)$ is centerless and $\operatorname{Out}\left(\pi_{1}(M)\right)$ is torsion-free. Moreover, $\operatorname{Aff}(M)$ is trivial if and only if both $L^{F}$ and $\operatorname{Out}\left(\pi_{1}(M)\right)$ are trivial.

Again there is an independent proof of this theorem, with the same argument as in Remark 5.4, but now referring to some well-known results presented in [KLR83].

Corollary 5.6. If the fundamental group of an infra-nilmanifold $M$ is complete, then $\operatorname{Aff}(M)$ is torsion-free. Conversely, if $\operatorname{Aff}(M)=\{1\}$, then $\pi_{1}(M)$ is complete.

Remark 5.7. In case $M$ is a flat Riemannian manifold ( $L$ is abelian), the condition $L^{F}=\{1\}$ is known to be equivalent to $Z\left(\pi_{1}(M)\right)=\{1\}$ ([HS86]), or $\operatorname{Aff}(M)=\{1\}$ if and only if $\pi_{1}(M)$ is complete. However, in general this is no longer valid and should be adapted. We refer to [IM96], where a generalised equivalent algebraic (and easier to verify) condition for $L^{F}=\{1\}$ is presented.

Let us now look for centerless (almost-)Bieberbach groups with torsion-free outer automorphism group. First, we present a low-dimensional example of a complete crystallographic group, unfortunately with torsion:

Example 5.8. Let $F$ be the subgroup of $\operatorname{GL}(2, \mathbb{Z})$ generated by $\left(\begin{array}{cc}1 & -1 \\ 1 & 0\end{array}\right)$ and $\left(\begin{array}{ll}0 & 1 \\ 1 & 0\end{array}\right)$, i.e. the dihedral group of order 12 . Let $E$ be the corresponding semi-direct product $\mathbb{Z}^{2} \rtimes F$, which is a 2-dimensional crystallographic (but not Bieberbach!) group fitting into an essential extension $1 \rightarrow \mathbb{Z}^{2} \rightarrow E \rightarrow \mathcal{D}_{6} \rightarrow 1$. One easily verifies that $F$ is self-normalising in $\mathrm{GL}(2, \mathbb{Z})$ and acts without fixed points on $\mathbb{Z}^{2}$. Moreover, we leave it as an exercise to check that $H^{1}\left(F, \mathbb{Z}^{2}\right)$ is also trivial and hence $E$ is complete (Proposition 4.4).

In fact, we will never find a complete Bieberbach group with $\mathcal{D}_{6}$ as holonomy group. Indeed, assume $1 \rightarrow N \rightarrow E \rightarrow F \rightarrow 1$ is an essential extension with abelian kernel $N$ and let the holonomy group $F$ be nilpotent or an extension of a cyclic group by a cyclic $p$-group. Then, asking that $H^{0}(F, N)$ and $H^{1}(F, N)$ vanish implies that $H^{2}(F, N)=\{1\}$ (Lemma 4.3). But then, $1 \rightarrow N \rightarrow E \rightarrow F \rightarrow 1$ splits and $E$ has torsion. In other words, because of Proposition 4.1.1, 
Proposition 5.9. There are no centerless Bieberbach groups with nilpotent holonomy or a holonomy group which is an extension of a cyclic group by a cyclic p-group, and with torsion-free outer automorphism group.

Or, equivalently,

Proposition 5.10. Each flat Riemannian manifold $M$ with nilpotent holonomy or a holonomy group which is an extension of a cyclic group by a cyclic p-group, admits a non-trivial finite effective group action as affine diffeomorphisms.

Because of some well-known results concerning primitive holonomy groups (finite groups which occur as holonomy group of a centerless Bieberbach group, see e.g. [HS86],[Szc90]), Proposition 5.9 enables us to decide that, up to dimension 5, there are no centerless Bieberbach groups with torsion-free outer automorphism group.

Remark 5.11. An analogous observation cannot be made for almost-Bieberbach groups. If $E$ fits into an essential extension $1 \rightarrow N \rightarrow E \rightarrow F \rightarrow 1$ and $\psi$ is the induced abstract kernel, then $H_{\psi}^{2}(F, Z(N))$ being trivial, in general, does not imply that $E$ has torsion (as we already mentioned in Remark 4.2).

Therefore, because of Lemma 4.5, we only know that

Proposition 5.12. There is always a non-trivial finite group of affine diffeomorphisms acting effectively on an infra-nilmanifold with a p-group as holonomy group.

Unfortunately, at this moment, no examples of complete (almost-)Bieberbach groups or centerless (almost-)Bieberbach groups with torsion-free outer automorphism group are known to the author. Therefore, we conjecture that infra-nilmanifolds always admit a periodic map:

Conjecture 5.13. All flat Riemannian manifolds and infra-nilmanifolds admit a non-trivial finite, effective group action as affine diffeomorphisms.

The analogue conjecture for infra-solvmanifolds of type $(\mathrm{R})$ is rejected by an example due to R. J. Koch ([CR71-2, p. 101]) of a 7-dimensional solvmanifold of type (R) admitting no periodic maps. On the other hand, the solvmanifold presented in Example 3.3 is not of type (R). If $\xi$ denotes the element of $N \rtimes \mathbb{Z}$ generating $\mathbb{Z}$, then the set of eigenvalues of $\mu(\xi)$ contains 1 and the eigenvalues of $\left(\begin{array}{ccc}0 & 0 & 1 \\ 1 & 0 & -1 \\ 0 & 1 & 0\end{array}\right)$ and $\left(\begin{array}{lll}1 & 0 & 1 \\ 1 & 0 & 0 \\ 0 & 1 & 0\end{array}\right)$ ([Dek97]). These are certainly not all positive real numbers as we have already noticed. Also the other examples of solvmanifolds without periodic maps presented in [CR71-2, pp. 95-96] are not of type (R).

\section{ACKNOWLEDGEMENT}

The author wishes to express his gratitude to his advisor Professor Paul Igodt, to Karel Dekimpe and to Andrzej Szczepański for their advice and for helpful conversations.

\section{REFERENCES}

[Aus60] Auslander, L. Bieberbach's Theorem on Space Groups and Discrete Uniform Subgroups of Lie Groups. Ann. of Math. (2), 1960, 71 (3), pp. 579-590. MR 22:12161

[Bro82] Brown, K. S. Cohomology of groups, volume 87 of Grad. Texts in Math. Springer-Verlag New York Inc., 1982. MR 83k:20002 
[CR71-1] Conner, P. E. and Raymond, F. Manifolds with few periodic homeomorphisms. Lect. Notes in Math. 299, Proceedings of the Second Conference on Compact Transformation Groups, Springer-Verlag, 1971, pp. 1-75. MR 50:11294

[CR71-2] Conner, P. E., Raymond, F., and Weinberger, P. J. Manifolds with no periodic maps. Lect. Notes in Math. 299, Proceedings of the Second Conference on Compact Transformation Groups, Springer-Verlag, 1971, pp. 81-108. MR 50:11295

[Dek96] Dekimpe, K. Almost-Bieberbach Groups: Affine and Polynomial Structures. Lect. Notes in Math. 1639, Springer-Verlag, 1996. CMP 98:04

[Dek97] Dekimpe, K. Determining the translation part of the fundamental group of an infrasolvmanifold of type (R). Math. Proc. Camb. Phil. Soc. 122 (1997), 515-524. CMP 97:17

[DIM93] Dekimpe, K., Igodt, P., and Malfait, W. On the Fitting subgroup of almost crystallographic groups. Bull. Soc. Math. Belg. Ser. B 45 (1993), 35-47. MR 95m:20056

[Gor71] Gorbacevič, V. V. Discrete subgroups of solvable Lie groups of type (E). Math. USSR Sbornik, 1971, $14 \mathrm{~N}^{\circ}$ 2, pp. 233-251. MR 44:6899

[Gor73] Gorbacevič, V. V. Lattices in solvable Lie groups and deformations of homogeneous spaces. Math. USSR Sbornik, 1973, 20 (2), pp. 249-266. MR 50:4816

[HS86] Hiller, H. and Sah, C.-H. Holonomy of flat manifolds with $b_{1}=0$. Quarterly J. of Math. Oxford (2), 1986, 37, pp. 177-187. MR 81:53073

[Hur36] Hurewicz, W. Beiträge zur Topologie der Deformationen. IV. Asphärische Räume. Nederl. Akad. Wetensch. Proc., 1936, 39, pp. 215-224.

[IM94] Igodt, P. and Malfait, W. Extensions realising a faithful abstract kernel and their automorphisms. Manuscripta Math., 1994, 84, pp. 135-161. MR 95k:20040

[IM96] Igodt, P. and Malfait, W. Representing the automorphism group of an almost crystallographic group. Proc. Amer. Math. Soc., 1996, 124 (2), pp. 331-340. MR 96d:20052

[KLR83] Kamishima, Y., Lee, K. B., and Raymond, F. The Seifert construction and its applications to infra-nilmanifolds. Quarterly J. of Math. Oxford (2), 1983, 34, pp. 433-452. MR 85k:57038

[Lee95] Lee, K. B. Infra-solvmanifolds of type (R). Quart. J. Math. Oxford (2), 1995, 46, pp. 185-195. MR 96k:57040

[LR81] Lee, K. B. and Raymond, F. Topological, affine and isometric actions on flat Riemannian manifolds. J. Differential geometry, 1981, 16 pp. 255-269. MR 84k:57027

[LR84] Lee, K. B. and Raymond, F. Geometric realization of group extensions by the Seifert construction. Contemporary Math. A. M. S., 1984, 33, pp. 353-411. MR 86h:57043

[LR89] Lee, K. B. and Raymond, F. Seifert Manifolds Modelled on Principal Bundles. Transf. Groups (Osaka,1987); Lect. Notes in Math., 1989, 1375 pp. 207-215. MR 90f:57041

[Mal51] Mal'cev, A. I. On a class of homogeneous spaces. Translations A.M.S., 1951, 39, pp. 1-33. MR 12:589e

[Mal96] Malfait, W. Nielsen's theorem for model aspherical manifolds. Manuscripta Math., 1996, 90, pp. 63-83. MR 97d:57001

[Par69] Parry, W. Ergodic properties of affine transformations and flows on nilmanifolds. Amer. J. Math., 1969, 91, pp. 757-771. MR 41:5595

[RT76] Raymond, F. and Tollefson, J. L. Closed 3-manifolds with no periodic maps. Trans. Amer. Math. Soc., 1976, 221, 2, pp. 403-418; 1982, 272, pp. 803-807. MR 54:3703; MR 83k:57033

[Rob80] Robinson, D. J. S. Infinite soluble groups with no outer automorphisms. Rend. Sem. Mat. Univ. Padova, 1980, 62, pp. 281-294. MR 81j:20051

[Seg83] Segal, D. Polycyclic Groups. Cambridge University Press, 1983. MR 85h:20003

[Szc90] Szczepański, A. Five dimensional Bieberbach groups with trivial center. Manuscripta Math., 1990, 68, pp. 191-208. MR 91h:57028

Department of Mathematics, Katholieke Universiteit Leuven Campus Kortrijk, Universitaire Campus, B-8500 Kortrijk, Belgium

E-mail address: Wim.Malfait@kulak.ac.be 